\title{
ONE-DIMENSIONAL AND TWO-DIMENSIONAL DYNAMICS OF CUBIC MAPS
}

\author{
DJELLIT ILHEM AND KARA AMEL
}

Received 20 December 2005; Accepted 24 March 2006

We concentrate on the dynamics of one-dimensional and two-dimensional cubic maps, it describes how complex behaviors can possibly arise as a system parameter changes. This is a large class of diffeomorphisms which provide a good starting point for understanding polynomial diffeomorphisms with constant Jacobian and equivalent to a composition of generalized Hénon maps. Due to the theoretical and practical difficulties involved in the study, computers will presumably play a role in such efforts.

Copyright (c) 2006 D. Ilhem and K. Amel. This is an open access article distributed under the Creative Commons Attribution License, which permits unrestricted use, distribution, and reproduction in any medium, provided the original work is properly cited.

\section{Introduction}

The study of discrete dynamical systems has been primarily limited to maps of an interval and/or diffeomorphisms. Diffeomorphisms of the plane exhibit some of the familiar properties of the quadratic Hénon map. The simplest maps are polynomial, and the simplest nontrivial diffeomorphism of the plane is the single Hénon map: $\left(x^{\prime}, y^{\prime}\right)=$ $\left(y+x^{2}+a, c x\right)$. This map is a two-dimensional discrete time system containing a single quadratic term as nonlinearity, and also known to display chaos for certain parameter values and initial conditions. Due to its simplicity, it has become a benchmark system and has been extensively studied because of its genericity, the complexity and richness of its dynamics, frequently used as an example for demonstrating schemes for analyzing and controlling chaotic behavior. Recently, different types of generalization of the standard Hénon map have been studied. In Dullin and Meiss [3] a two-dimensional map is treated whose single polynomial term generalizes the quadratic nonlinearity of the Hénon map.

The set of polynomial maps with polynomial inverse is called the "affine Cremona group"- -very dynamically interesting maps. The structure of this group is well known and understood for two-dimensional case; Friedland and Milnor [5] showed that any map in this group of polynomial diffeomorphisms is conjugate to a composition of generalized Hénon maps: $\left(x^{\prime}, y^{\prime}\right)=(y+p(x), c x)$; a map with a constant and nonzero Jacobian 
and where $p(x)$ is a polynomial. It follows that any composition of Hénon maps has an inverse which is polynomial. The latter generalized Hénon maps (GHM) appear to be of particular interest since they offer to study higher-dimensional chaos where the generating equation is simple.

On the other hand, a rigorous theory of the dynamics of noninvertible maps of the plane has been recently developed. Many of the analytical techniques and theory applied to diffeomorphisms are either unavailable or limited in the context of two-dimensional endomorphisms of the plane. Principal method is the method of critical curves which provides researchers in this field with analytical and experimental tools. Several papers have shown the importance of critical curves in the bifurcations of basins, for example, the transition type "simply connected basin $\leftrightarrow$ nonconnected basin" as Gumowski and Mira [8] who have developed the role of critical curves in bifurcations, Barugola and Cathala [1] and Gardini $[6,7]$ have studied bifurcations of type "simply connected basin $\leftrightarrow$ multiply connected basin." These basic bifurcations result from the contact of a basin boundary with a critical curve segment of an attracting set, such a bifurcation leads either to the chaotic area destruction, or to a sudden and important modification of the area.

Many of chaotic motions that are observed in dynamical systems are intimately associated with the presence of transversal homoclinic points of maps. Contact bifurcations may correspond to homoclinic and heteroclinic bifurcations, and critical curves are useful for interpreting such problems. Bifurcations by homoclinic and heteroclinic contact have been presented in [7] for the one-dimensional case, in [6,7] it is proved that some contact bifurcations correspond to homoclinic bifurcations in the case involving a repelling node or focus and other examples of homoclinic orbits of saddles.

This work presents research in the study of cubic invertible and noninvertible maps of the plane that carried out some techniques and numerical simulations. This set is of fundamental importance in dynamical systems. Two main tools are used by developing the method of critical curves and incorporating the polynomial maps theory: two wellestablished theories. Due to the theoretical and practical difficulties involved in the study, computers will presumably play a role in such efforts. Our aim is to use computers not only for inspiration, but to perform rigorous mathematical proofs.

First we recall the bifurcation structure of the one-dimensional endomorphism of the cubic model

$$
T_{o}(x)=x^{3}+(a+1) x+b .
$$

The global organization of bifurcation in this map is proposed of "box-within-a-box" fractal type in a foliated parameter plane [9], where the basic elements of this structure are cross-road areas, spring areas, and saddle areas (see [11]) which are related to three dispositions of fold and flip bifurcations curves around a cusp point.

We emphasize that our aim is to study simply the cubic maps. We consider an imbedding of (1.1), which is a one-dimensional noninvertible map, into a two-dimensional diffeomorphism. This diffeomorphism can be considered as a model which only gives rise to a set of bifurcations with a fractal structure, and does not give rise to birth of cyclic invariant curves. We study this diffeomorphism in dependance of at least three parameters, using both analytic perturbation theory and numerical methods. The dynamics 


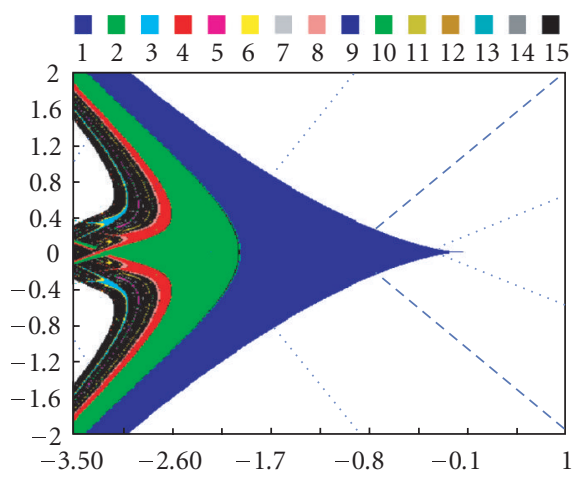

(a) Bifurcation structure $c=0$

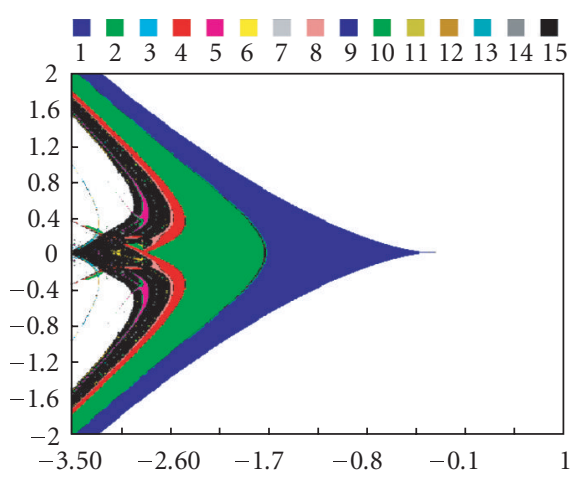

(b) Bifurcation structure $c=0.25$

Figure 1.1

involves various transitions by bifurcations. Perturbation theory here reveals periodicity and quasiperiodicity, parametrically organized by embedded boxes structure. Outside these parameter regimes the perturbation becomes larger and bifurcations complicate the dynamics, often involving chaos.

The planar diffeomorphism is the following:

$$
\cdot T_{1}:\left\{\begin{array}{l}
x_{n+1}=x_{n}^{3}+(a+1) x_{n}+b+y_{n} \\
y_{n+1}=c x_{n}
\end{array}\right.
$$

where $x, y$ are real variables, $a, b$, and $c$ are real parameters. $T_{1}$ has a constant Jacobian determinant det $J=-c$.

For $c=0,(1.2)$ is the one-dimensional endomorphism (1.1) embedded into the twodimensional diffeomorphism (1.2), and has some symmetry property and possesses at most three fixed points depending upon the parameter values. We describe a specific family of bifurcations in a region of real-parameter $(a, b)$ plane for which the mappings were expected to have simple dynamics. We compute the first few bifurcation curves in this family and we study the bifurcation diagram which consists of these bifurcation curves in the parameter $(a, b)$ plane together with representative phase portraits. Figures 1.1(a), 1.1(b) present information on stability region for the fixed point (blue domain), and the existence region for attracting cycles of order $k$ exists $(k \leq 14)$. The black regions $(k=15)$ correspond to the existence of bounded iterated sequences. These figures are typical of maps with dominating cubic terms, analogous bifurcation diagrams have already been obtained for systems with nonlinearity given by hyperbolic tangency $[4,12]$. We can recognize on the diagram period doubling bifurcation, saddle area and spring area [11]. The bifurcation structure is a cubic "box-within-a-box" type, as is well known infinitely many periodic are opened by fold bifurcations and are closed by homoclinic bifurcations by the intriguing "box-within-a-box" bifurcation structure. When $c \neq 0$, there is an interesting passage from the one-dimensional endomorphism to the two-dimensional diffeomorphism, and then some properties are automatically deduced. 
4 One-dimensional and two-dimensional dynamics of cubic maps

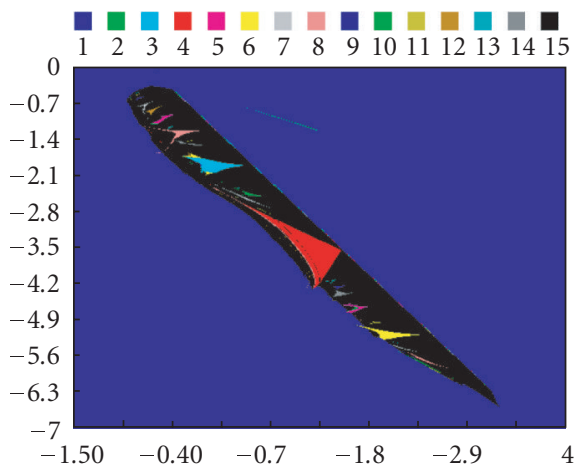

(a)

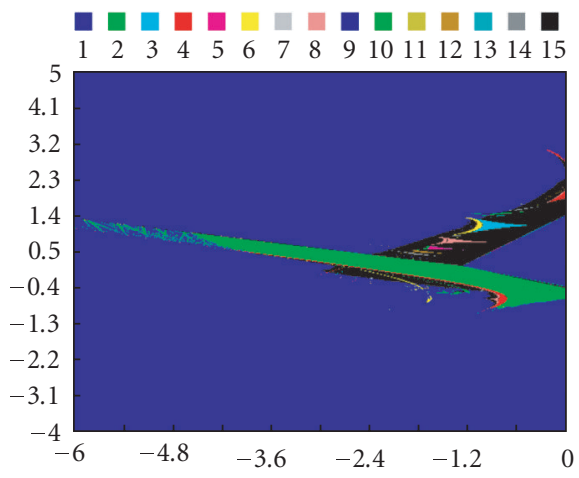

(c)

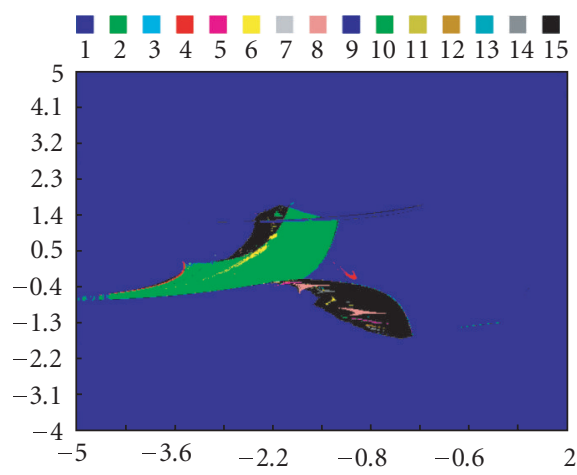

(b)

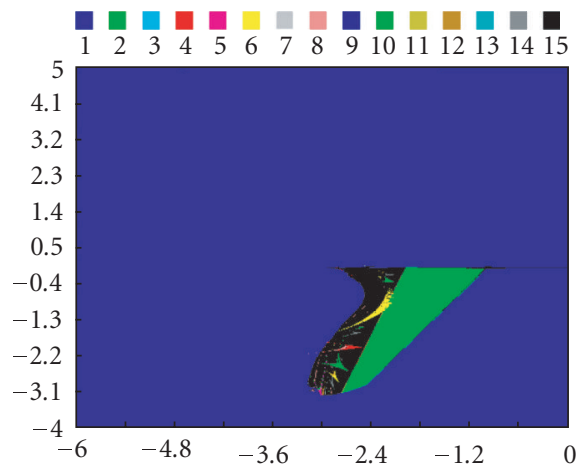

(d)

Figure 1.2. Bifurcation diagrams, (a) in $(a+1, c)$ plane for $h=0.9, d=-1$, (b) in $(a+1, d)$ plane for $h=0.9, c=-0.7$, (c) in $(a+1, h)$ plane for $c=d=-0.7$, (d) $c=d=1$.

When $c=0, T_{0}$ is called of $\left(Z_{1}-Z_{3}-Z_{1}\right)$ type (in means of Mira [11]). Such maps constitute a class having the simplest properties, maps of $\left(Z_{1}-Z_{3}-Z_{1}\right)$ type are generally characterized by the existence of one, three, and one rank-one preimages. Their study is indispensable before considering more complex types, which locally may have the $\left(Z_{1}-\right.$ $\left.Z_{3}-Z_{1}\right)$ properties.

Let us consider now the noninvertible map $T_{2}$ defined by

$$
T_{2}:\left\{\begin{array}{l}
x_{n+1}=\epsilon x_{n}^{3}+(a+1) x_{n}+b+h y_{n} \\
y_{n+1}=c x_{n}+d y_{n}
\end{array}\right.
$$

where $d, h$ are real parameters and $\epsilon= \pm 1$. Figure 1.2 gives the different bifurcation diagrams for $T_{2}$, where the existence regions of attracting sets of period $k$ are shown, the periods are indicated by colored squares. This scanning gives a first idea and a global view of the different kinds of attracting sets and their parameter dependant bifurcations, which show up when we study this map in its dynamic context and even lead to chaos. 
For this map we use the critical curve notion which is an important mathematical tool used to study bifurcations. Mira [11] provides an entry into certain areas on noninvertible maps and the role of such curve in bifurcations basin. We define the critical curve $L C$ of an endomorphism $T$ in the plane $\mathbb{R}^{2}$ (in means of Mira [11]) as the geometrical locus of points $x$ having at least two coincident preimages of first rank. We determine the locus of the initial line, said to have the rank -1 , and denoted by $L C_{-1}$, when $T_{2}$ is differentiable, by taking the Jacobian determinant of $T_{2}$ to be equal to zero $\left(\operatorname{det}\left(\operatorname{DT}_{2}(x, y)\right)=0\right)$. A critical line $L C$ is constituted of one or several branches. These branches separate the plane in open regions, where all points of a region have the same number of first rank antecedents. A train of critical curves, tangent to each other, bounds the absorbing area for the attractor.

When $h=c=0$ we have the composition $r \circ \Upsilon$ of a classical discrete-time map modeled by the iteration of the two-dimensional map

$$
\Upsilon:\left\{\begin{array}{l}
x^{\prime}=r_{1}(y), \\
y^{\prime}=r_{2}(x),
\end{array}\right.
$$

and the reflection $r(x, y)=(y, x)$.

$r_{1}$ and $r_{2}$ are real functions defined in $X \subset \mathbb{R}$ and $Y \subset \mathbb{R}$, respectively (so that $Y$ is defined in the rectangle $X \times Y$ ). In a paper by Rand (see [14]), it is proved that quite complex dynamics, with periodic and chaotic trajectories, can emerge from the iteration of $\Upsilon$. Peculiar properties of this kind of map can be deduced from the properties of one-dimensional maps obtained by the composition of the real functions, while peculiar properties of the bifurcations are given in Lupini et al. [10]. Bischi et al. in [2] showed that, in general, map of the form $\Upsilon$ is characterized by multistability.

In our case, these real functions are expressed by $r_{1}(y)=y$ and $r_{2}(x)=x^{3}+(a+1) x+$ $b$, where $c=h=0$.

Since $T_{2}$ is noninvertible, so global analyses which use the theory of critical lines cited above apply. Noninvertibility means that there exists a set in phase plane where the Jacobian determinat of the map vanishes. The forward image of this set is called line $L C$. The existence of such a set brings a specific character into bifurcation scenarios, shapes of attracting sets and their basins of attraction, and so forth, different from those known for invertible maps.

$T_{2}$ with $h=\epsilon=1$ is of type $\left(Z_{1}-Z_{3}-Z_{1}\right)$, whose critical curves $L C_{-1}, L C_{-1}^{\prime}, L C, L C^{\prime}$ are given here by

$$
\begin{gathered}
\left(L C_{-1}: x=+\sqrt{\frac{c}{3 d}-\frac{a+1}{3}}, L C_{-1}^{\prime}: x=-\sqrt{\frac{c}{3 d}-\frac{a+1}{3}}\right) \\
\left(\begin{array}{l}
L C: y=d \cdot x-d \cdot x_{0}^{3}-(a \cdot d+d-c) x_{0}-d \cdot b \\
L C^{\prime}: y=d \cdot x+d \cdot x_{0}^{3}+(a \cdot d+d-c) x_{0}-d \cdot b
\end{array}\right) \quad \text { with } x_{0}=\sqrt{\frac{c}{3 d}-\frac{a+1}{3}} .
\end{gathered}
$$


The critical curves of rank $k$ are defined as $L C_{k}=T^{k}(L C)$ and $L C_{k}^{\prime}=T^{k}\left(L C^{\prime}\right)$. The successive images of the critical curves define an absorbing area $A$ such that if some trajectory enters $A$, it can never leave this area, and such that there exists a neighborhood $U(A)$ whose points will be mapped into $A$ in a finite number of iterations. The boundary of this area is made up by portions of the images of $L C$ and $L C^{\prime}$.

Figure 1.2 presents the parameter regions of existence of attracting cycles and regions of divergence. The well-known "Arnold tongues" are clearly seen, and regions of coexistence of several attracting cycles can be observed. These occur where there are overlapping tongues associated with cycles of different periods. What will happen with the phase plane of $T_{2}$ if we fix the parameters values inside some tongues of periodicity? This question is closely related to the problem of the breakdown of a two-dimensional torus, which is well studied for diffeomorphisms, but is a more complicated task for noninvertible maps. This paper intends to give such a study, and to consider the different maps. Therefore, it is structured in the following way. In Section 2 we introduce the language used in $[5,11]$ to analyze these maps, and we note basic definitions and facts about Hénon maps. Section 3 gives some results on basin structures of invertible cubic maps and their bifurcations. Section 4 illustrates properties of bifurcations of noninvertible cubic maps.

\section{Definitions and fundamental properties}

In this section, we define precisely a number of dynamical properties commonly associated with invertible polynomial maps, chaotic area, contact and homoclinic bifurcations, and some properties of increasing complexity that try to highlight the important concepts of nonlinear maps. These are the properties which will appear in our work.

The polynomial map $T$ of the plane has the form

$$
\left(x^{\prime}, y^{\prime}\right)=T(x, y)=(f(x, y ; \lambda), g(x, y ; \lambda))
$$

where $f$ and $g$ are polynomials in $x, y$ and $\lambda$ is a real parameter-vector. The Jacobian determinant is defined as

$$
\operatorname{det} J(f, g)=\operatorname{det} T(x, y)=\frac{\partial f}{\partial x} \frac{\partial g}{\partial y}-\frac{\partial f}{\partial y} \frac{\partial g}{\partial x}
$$

2.1. General properties. We assume that a closed and invariant set $A$ is called an attracting set if some neighborhood $U$ of $A$ may exist such that $T(U) \subset U$, and $T^{n}(x, y) \rightarrow A$ as $n \rightarrow \infty$ for all $(x, y) \in U$. The set $\mathbb{B}=\cup_{n \geq 0} T^{-n}(U)$ is called the total basin of $A$.

Definition 2.1. A chaotic area $A$ is an invariant absorbing area $(T(A)=A)$ that exhibits chaotic dynamics, and the points of which give rise to iterated sequences having the property of sensitivity to initial conditions.

Definition 2.2. $\lambda=\lambda^{*}$ is a bifurcation of contact of $A$ if a contact between the frontier of $A$ and the frontier of its basin of attraction takes place. 
Proposition 2.3 [13]. When a bifurcation of contact of a chaotic area $A$ arises for a value $\lambda=\lambda^{*}$, the crossing of this value leads to the destruction of $A$ or to a qualitative modification of properties of $A$.

Definition 2.4. Let $T$ be an endomorphism of $\mathbb{R}^{2}$ depending on a parameter $\lambda$ and let $S$ be a saddle point of $T$. A homoclinic bifurcation takes place if for a value $\lambda=\lambda^{*}$, there is apparition (or disappearance) of an infinity of homoclinic orbits.

2.2. Generalized Hénon map properties. First we recall the dynamics of diffeomorphism of the plane and let us take $T$ of the form

$$
T(x, y)=(y+p(x), c x)
$$

with $p(x)$ a degree 3 polynomial then $T$ is conjugate to a generalized Hénon map. We know some results which enable us to detect, predict, determine cycles and fixed points, and locate bifurcation curves in parameter space.

It is sufficient to consider the case $|c| \leq 1$, since the inverse of a generalized Hénon map with $|c|>1$ is conjugate to a generalized Hénon map with $|c|<1$ under the reflection $r(x, y)=(y, x)$, and $r \circ T^{-1} \circ r=(y-p(x / c), x / c)$.

Since $p(x)$ is a degree odd, then $T$ has a symmetry $s^{-1} \circ T^{-1} \circ s=T, s(x, y)=(-x,-y)$ when $p(-x)=-p(x)$.

Fixed point $\left(x_{*}, y_{*}\right)$ of $T$ satisfies $y_{*}=c x_{*}$, and $(1-c) x_{*}=p\left(x_{*}\right)$, so that $x_{*}$ is a root of the polynomial $q(x)=(c-1) x+p(x)$, thus all fixed points are located on the line $y-c x=0$ in the plane.

The stability of these fixed points is determined by the Jacobian $J=\left(\begin{array}{cc}p^{\prime}(x) & 1 \\ c & 0\end{array}\right)$ which has trace $\operatorname{tr}=p^{\prime}(x)$ and determinant $\operatorname{det} J=-c$. The fixed point is stable if its parameters belong to the interior of the triangle $|-\operatorname{tr}|-1 \leq \operatorname{det} J \leq 1$ in $(\operatorname{tr}, \operatorname{det} J)$ space, because $J$ is a contraction, so it is necessary that $|-\operatorname{tr}| \leq 1+\operatorname{det} J$ for stability.

It is easy to verify according to $[3,5]$ that any generalized Hénon map can have bounded orbits only when there are fixed points.

Theorem 2.5 [3]. Suppose a generalized Hénon map has no fixed points, then every orbit is unbounded.

Proof. Suppose that $T$ has no fixed points, then the polynomial $q(x)=p(x)+c x-x$ is either positive or negative for all $x$. In the first case $q(x)$ is positive, consider $d(x, y)=$ $x+y$ then $d\left(x^{\prime}, y^{\prime}\right)=d(x, y)+q(x)$ creases monotonically and must be unbounded. In the other case $q(x)$ is negative, $d\left(x^{\prime}, y^{\prime}\right)$ decreases monotonically, and then in either case there are no bounded orbits.

When there are fixed points, we can find a box that contains all these bounded orbits.

Theorem 2.6 [3]. Every bounded orbit of a generalized Hénon map is contained in the box $\{(x, y):|x| \leq M|y| \leq|c| M\}$, where $M$ is the largest of the absolute values of $\mid p(x)-(1+$ $|c|) x \mid$. 


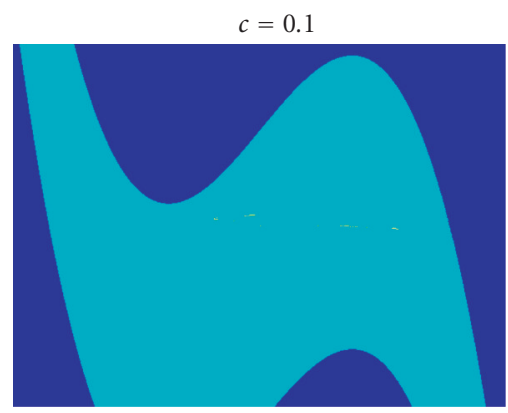

(a) $c=0.1$

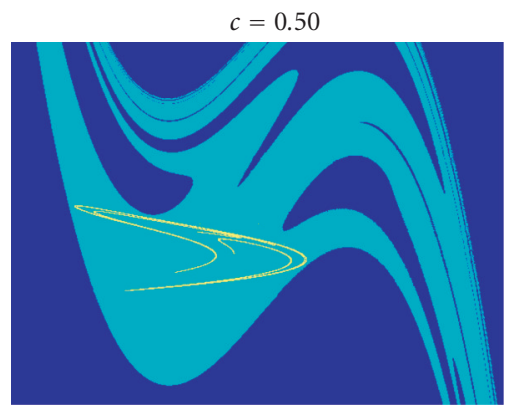

(c) $c=0.50$

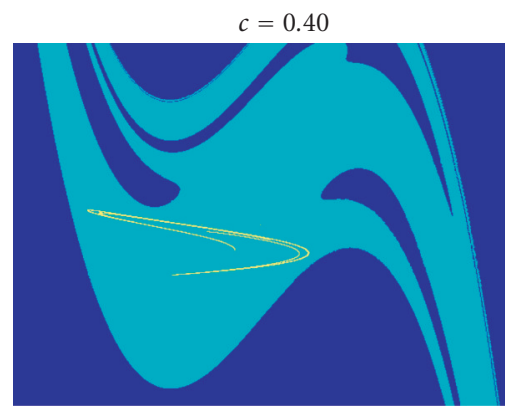

(b) $c=0.40$

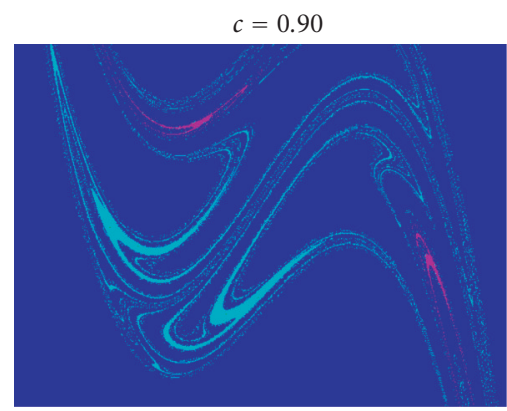

(d) $c=0.90$

Figure 3.1. Destruction of the chaotic area and its basin after a contact bifurcation.

Proof. See [3], more generally the polynomial determining $M$ is the same as that for the fixed points, up to the absolute value signs.

\section{Basins and attractors for the diffeomorphism}

In this section we give a sketch on bifurcations which give rise to fractalization of attractor and its basin boundary. The main purpose of this section is to give some results on basin structure of invertible maps and their bifurcations. Especially, we explain basin bifurcations. These basic bifurcations result from the contact of a basin boundary with a chaotic attractor, such a bifurcation leads either to the chaotic area destruction, or a sudden and important modification of this area, some properties of maps were presented in $[1,11]$ from the point of view of fixed point bifurcations, with chaotic attractors and absorbing area bifurcations. We choose $a=-2.67, b=0.47, h=1$ and we vary $c$.

The following figures show the corresponding basin structure of $T_{1}$. Figures 3.1(a), 3.1(b), 3.1(c), and 3.1(d) represent the existing attractors (order $k$ cycles, invariant curves, or chaotic attractors) and their basins. The evolution of attractors and their basins is given directly in figures, the parameters $(a, b)$ have been chosen constant and $c$ varies. There exist several ways in which a dynamical system can become chaotic, of which the 
period-doubling route to chaos is the best known and identified here. We can see also that the bifurcation, which is put in evidence, corresponds to a limit contact between the basin boundary $\partial \mathbb{B}$ and the chaotic attractor. A further increase of the parameter $c$ causes a contact between these two boundaries which marks the destruction of the basin of attraction. As argued in [11], we can see the ghost of this area destroyed at the contact.

\section{Basin bifurcations for the endomorphism}

In this section we consider the two-dimensional endomorphisms $T_{2}$, of which the $L C$ is constituted of two distinct branches, separates the phase plane in three open regions $Z_{1}^{1}$, $Z_{3}$, and $Z_{1}^{2}, Z_{3}$ is the place of points having three preimages of distinct first rank $Z_{1}^{1}$, and $Z_{1}^{2}$ is the one of points having only one antecedent. These applications are called of type $Z_{1}-Z_{3}-Z_{1}$.

We denote, respectively, by $\mathbb{B}_{0}$ and $\mathbb{B}$ immediate basin of attraction and total attraction basin of an attractor. The region not containing the attractor is called an island. It is interesting to know when basins are connected or nonconnected. The creation of holes inside the basin is considered as a bifurcation, that means a qualitative change in the system behavior. This can be explained on the basis of the critical curves properties and it is the same for the chaotic attractors and their bifurcations.

The publication [1] deals with basin bifurcation, and the fractalization of the domain $\mathbb{B}$, but using other techniques. These bifurcations are called contact bifurcations. With noninvertible maps, contact bifurcations; that is, contact between the boundary of a basin and the critical curve, are at the origin of various situations giving rise to heteroclinic orbits and heteroclinic connections.

Different types of fractalization of basin boundary occur, a part of them resulting from a phenomenon of islands aggregation for nonconnected basins presented in [11].

Proposition 4.1 [11]. Consider an endomorphism T depending on a parameter $\lambda$. If the connected components number of $\mathbb{B} \cap L C$ changes when $\lambda$ crosses bifurcation value $\lambda^{*}$, then the basin $\mathbb{B}$ may undergo a qualitative change of one of the following types:

(a) connected basin $\leftrightarrow$ nonconnected basin (when the number of connected components of $\mathbb{B}_{0} \cap L C$ changes);

(b) simply connected basin $\leftrightarrow$ multiply connected basin (when the number of connected components of $\mathbb{B}_{0} \cap L C$ changes);

(c) modification of the number of lakes in $\mathbb{B}$ or new arborescent sequence of islands;

(d) destruction of a chaotic area.

These bifurcations essentially correspond to an interaction of stable manifold associated with a saddle point or a saddle cycle (they generally constitute the boundary of the basin of attraction of an area or a chaotic attractor) with the critical lines (which constitute the boundary of the area or the chaotic attractor).

For fixed parameter values, we plot the attraction basin of an attractor. When there exist several attracting sets, it is possible to define a global basin, that means the set of initial conditions giving rise to bounded iterated sequences, independently of the fact that they converge to one attractor or another. We choose $c=-1, b=0, d=-1.498$, $h=\epsilon=1, a$ varying. 


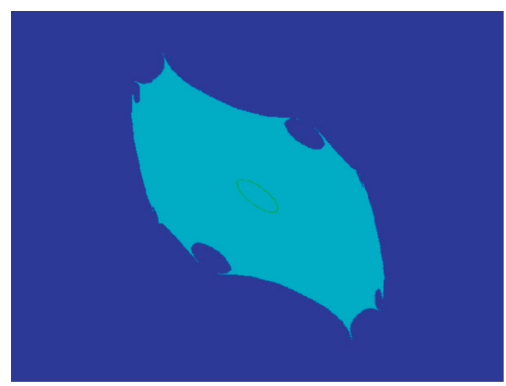

(a) $a=-1 ;(x, y) \in[-2,2] \times[-2,2]$. Tongues appear on basin boundary. Invariant closed curve shows stability loss of the fixed point

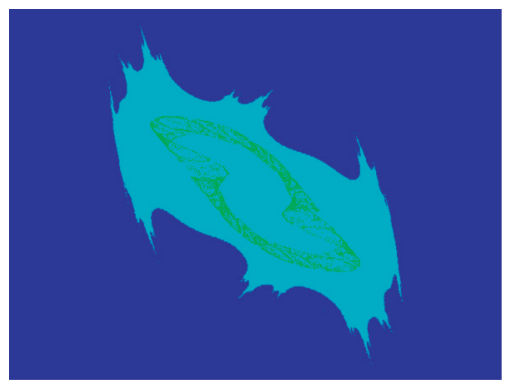

(c) $\quad a=-0.6 ; \quad(x, y) \in[-2,2] \times$ $[-2,2]$. Chaotic attractor

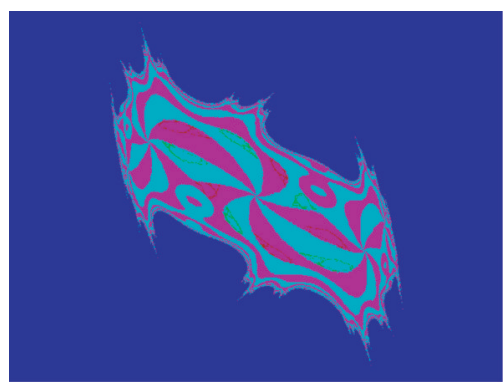

(b) $a=-0.59$. Bistability; we have coexistence of two invariant curves of order 5

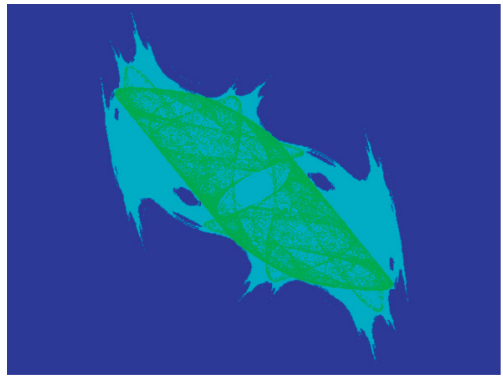

(d) $a=-0.79 ; \quad(x, y) \in[-2,2] \times$ $[-2,2]$. Invariant absorbing area, obtained by iteration of $L C$, including the Milnor attractor

Figure 4.1

These models were proposed by more than a dozen authors for a wide variety of reasons. They all incorporate nonlinear terms particularly or other quadratic nonlinearities. Surprisingly, in most cases, the basin of attraction undergoes changes which present a real interest and where we can see a large richness of the bifurcations situations and new structures of basins and invariant sets. The characteristic of these maps gives rise to specific singularities which induce important effects on the geometrical and dynamical properties of the phase plane. Some of these results have been shown before for the Hénon maps (see Figure 4.1); others are new even in this restricted context (see Figures 4.2 and 4.4). Some require delicate proofs and others follow with a bit of work from the general theory cited here.

We choose $a=-1, b=0, h=0.9, d=-0.9$ and we vary $c$. Figure 4.3 shows basins of two coexisting attractors.

For $\epsilon=-1, a=-3, b=0, h=-0.01, c=0.01$, and $d=1$, we show the shape of chaotic attractor transformed into a relaxation cycle (Figure 4.4), that is, a combination 


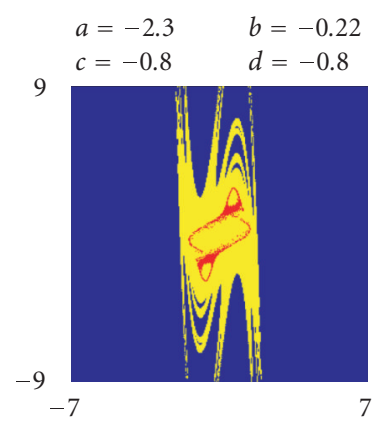

(a) Other chaotic attractor

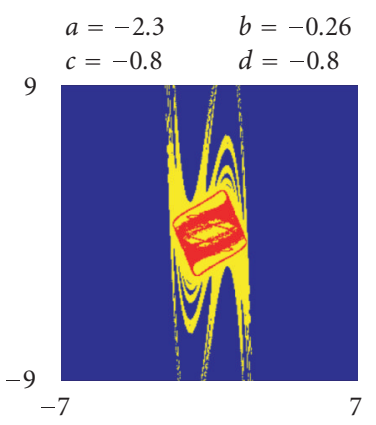

(b) Other chaotic attractor

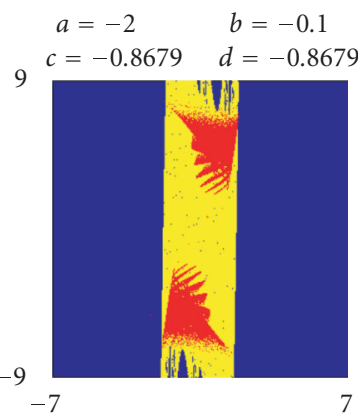

(c) The period-2 chaotic attractor

Figure 4.2

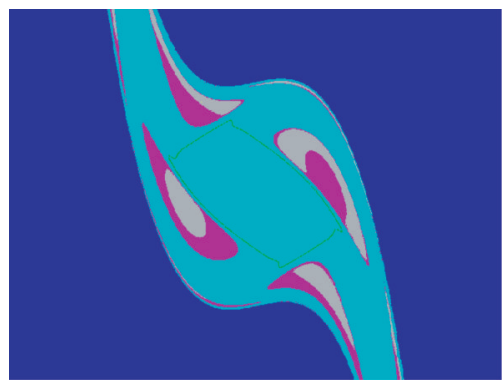

(a) $c=-1.56$

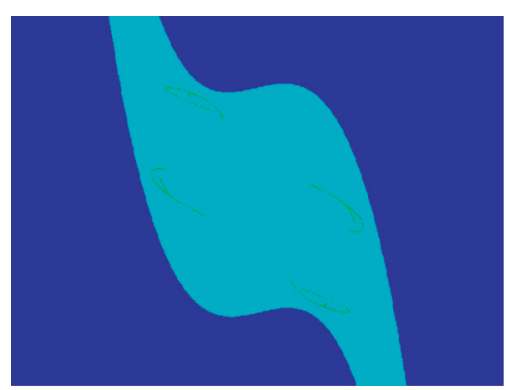

(c) $c=-1.67$

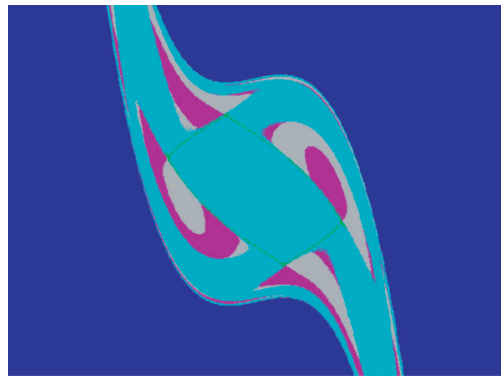

(b) $c=-1.58$

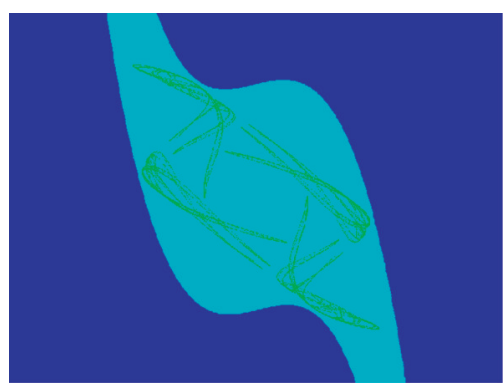

(d) $c=-1.78$

Figure 4.3. (a) The colors correspond to the basins of the two existing attractors: the invariant closed curve and a 4-periodic orbit. (b) The closed curve grows in size (c) and (d) for $c=-1.67$ to -1.78 the period-4 chaotic attractor near the boundary crises.

of smooth movement and sudden jumps, further with two inserted copies of the bifurcation diagram. It is a cycle where the relaxation jump enters a chaotic zone from which the cycle again simplifies through a period halving route to order. This is the disordered 


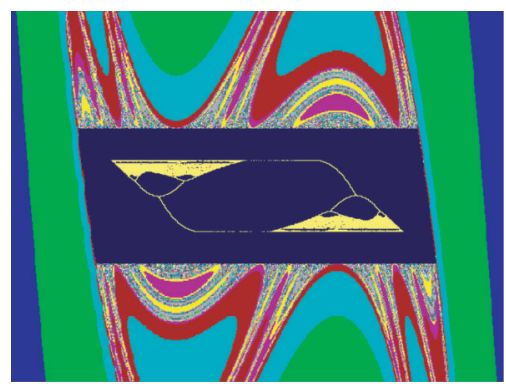

(a)

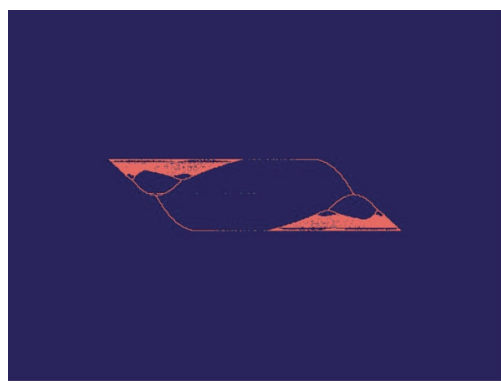

(b)

Figure 4.4. Emergence of the relaxation cycle and its basin.

phases of the cycle which occur in the transitions between phases of prosperity and depression.

\section{Conclusion}

In this paper, specific bifurcations arising in maps of cubic type have been studied. These bifurcations concern the evolution of chaotic attractors, which appear or disappear by contact bifurcations, with their own basin boundary. In such maps, chaotic attractors can be issued of a succession of period doubling bifurcations $[5,11]$. The dynamical structure is at once complicated and simple, because it is infinite in quantity. These maps have an extremely rich dynamical structure, the complicated internal structure gives different scenarios of bifurcations.

\section{References}

[1] A. Barugola and J. C. Cathala, An extension of the notion of chaotic area in two-dimensional endomorphisms, Proceedings of the European Conference on Iteration Theory (ECIT '92), Batschuns, September 1992.

[2] G. I. Bischi, C. Mammana, and L. Gardini, Multistability and cyclic attractors in duopoly games, Chaos, Solitons and Fractals 11 (2000), no. 4, 543-564.

[3] H. R. Dullin and J. D. Meiss, Generalized Hénon maps: the cubic diffeomorphisms of the plane, Physica D. Nonlinear Phenomena 143 (2000), no. 1-4, 262-289.

[4] D. Fournier-Prunaret, Chaotic attractors in transmission system, Proceedings of the International Symposium on Nonlinear Theory and Its Applications (NOLTA '98), vol. 2, Crans-Montana, September 1998, pp. 735-738.

[5] S. Friedland and J. Milnor, Dynamical properties of plane polynomial automorphisms, Ergodic Theory and Dynamical Systems 9 (1989), no. 1, 67-99.

[6] L. Gardini, Some global bifurcations of two-dimensional endomorphisms by use of critical lines, Nonlinear Analysis. Theory, Methods \& Applications 18 (1992), no. 4, 361-399.

[7] __ Homoclinic bifurcations in n-dimensional endomorphisms, due to expanding periodic points, Nonlinear Analysis. Theory, Methods \& Applications 23 (1994), no. 8, 1039-1089.

[8] I. Gumowski and C. Mira, Accumulations de bifurcations dans une récurrence, Comptes Rendus de l'Académie des Sciences. Paris, Série A-B 281 (1975), no. 1, Aii, A45-A48.

[9] _ Dynamique Chaotique, Cepadues Éditions, Toulouse, 1980. 
[10] R. Lupini, S. Lenci, and L. Gardini, Bifurcations and multistability in a class of two-dimensional endomorphisms, Nonlinear Analysis. Theory, Methods \& Applications 28 (1997), no. 1, 61-85.

[11] C. Mira, Chaotic Dynamics, World Scientific, Singapore, 1989.

[12] C. Mira, D. Fournier-Prunaret, L. Gardini, H. Kawakami, and J.-C. Cathala, Basin bifurcations of two-dimensional noninvertible maps: fractalization of basins, International Journal of Bifurcation and Chaos in Applied Sciences and Engineering 4 (1994), no. 2, 343-381.

[13] C. Mira, L. Gardini, A. Barugola, and J.-C. Cathala, Chaotic Dynamics in Two-Dimensional Noninvertible Maps, World Scientific Series on Nonlinear Science. Series A: Monographs and Treatises, vol. 20, World Scientific, New Jersey, 1996.

[14] D. Rand, Exotic phenomena in games and duopoly models, Journal of Mathematical Economics 5 (1978), no. 2, 173-184.

Djellit Ilhem: Department of Mathematics, University of Annaba, BP 1223000 Annaba, Algeria

E-mail address: i_djellit@hotmail.com

Kara Amel: Department of Physics, University of Sétif, 19000 Sétif, Algeria

E-mail address: akhachemi@yahoo.fr 


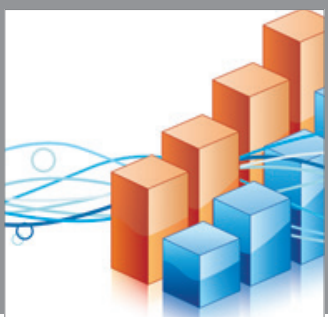

Advances in

Operations Research

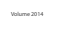

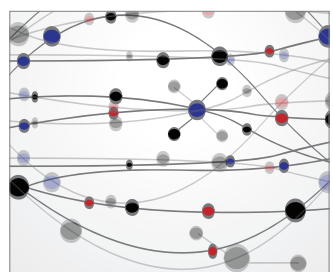

\section{The Scientific} World Journal
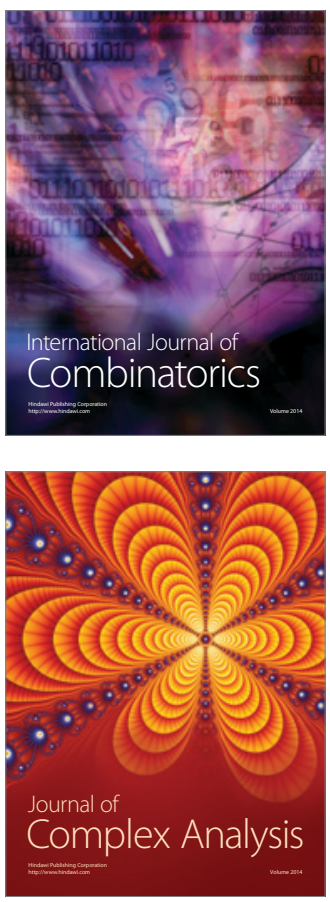

International Journal of

Mathematics and

Mathematical

Sciences
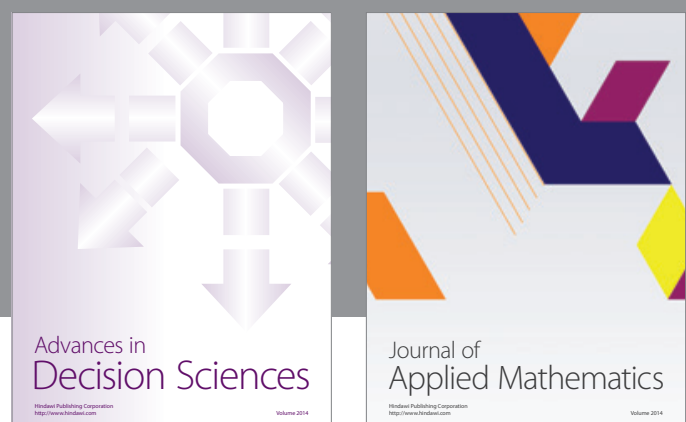

Journal of

Applied Mathematics
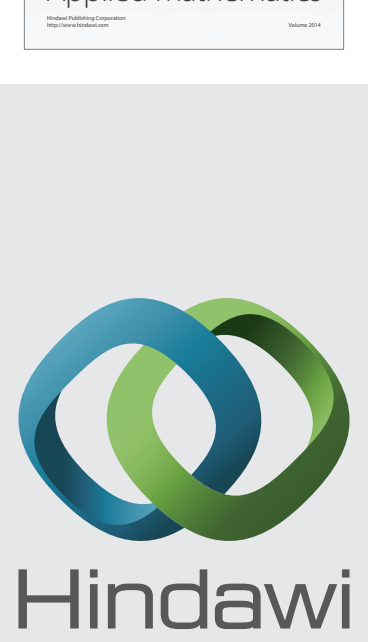

Submit your manuscripts at http://www.hindawi.com
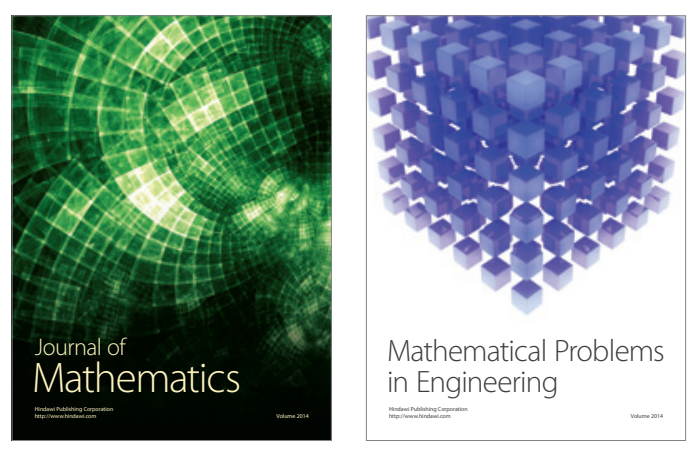

Mathematical Problems in Engineering
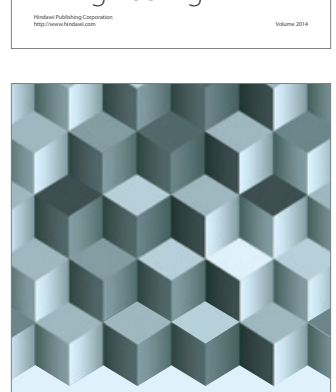

Journal of

Function Spaces
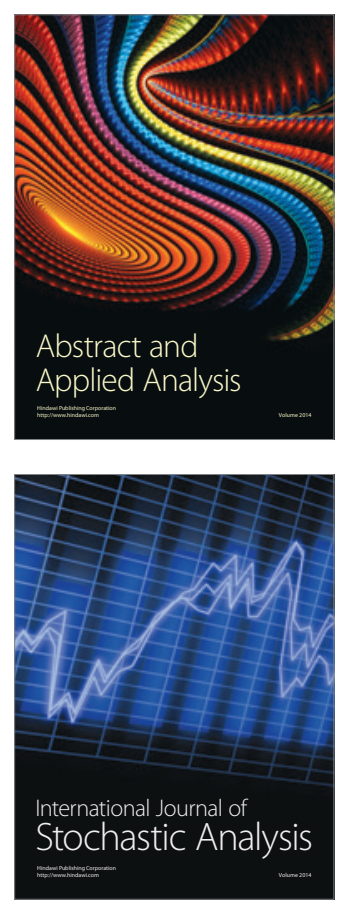

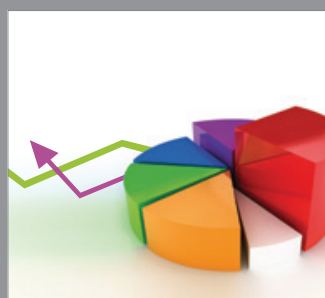

ournal of

Probability and Statistics

Promensencen
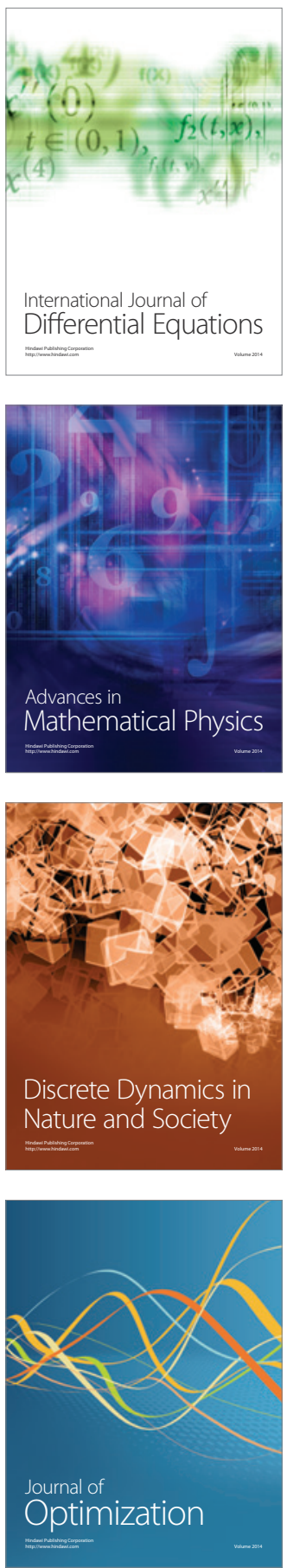